\title{
Влияние одноосной деформации в направлении [110] на релаксацию состояний мелких доноров мышьяка в германии
}

\author{
(C) В.В. Цыпленков, В.Н. Шастин
}

Институт ффизики микроструктур Российской академии наук, 603950 Нижний Новгород, Россия

E-mail: Tsyplenkov1@yandex.ru

Поступила в Редакцию 15 апреля 2020 г.

В окончательной редакции 21 апреля 2020 г.

Принята к публикации 21 апреля 2020 г.

\begin{abstract}
Проведен анализ скоростей релаксации состояний доноров мышьяка в германии при взаимодействии с акустическими фононами в зависимости от одноосной деформации сжатия кристалла в направлении [110] при низких температурах $(<10 \mathrm{~K})$. Показано, что при оптическом возбуждении происходит формирование инверсной заселенности уровней донора, зависящей от величины деформации кристалла, что дает основания предположить возможность эффекта стимулированного излучения в ТГц диапазоне частот на внутрицентровых переходах мелких доноров мышьяка при их оптическом возбуждении. Показано, что одноосная деформация в направлении [110] может приводить к переключению рабочего перехода и, следовательно, к изменению частоты стимулированного излучения.
\end{abstract}

Ключевые слова: германий, мелкие доноры, релаксация, излучение фононов.

DOI: 10.21883/FTP.2020.09.49832.24

\section{1. Введение}

Поиск новых компактных источников когерентного излучения в ТГц диапазоне в настоящее время остается актуальной задачей. Особенный интерес вызывают полупроводниковые источники на основе кремния и германия, что обусловлено развитостью соответствующей технологии. Ранее теоретически было показано, что в кристалле германия, легированном донорами мышьяка (As), при оптическом возбуждении среды формируется инверсная населенность уровней доноров, что приводит к возможности генерации стимулированного излучения в диапазоне частот ( 1.2-2 ТГц) [1]. Возможность формирования инверсной населенности также косвенно подтверждена экспериментально на основе измерения времен релаксации возбужденных состояний доноров мышьяка [2]. Несмотря на наличие в этом диапазоне других источников излучения, появление источника на основе объемного Ge было бы очень полезным. В настоящей работе проведены вычисления скоростей релаксации возбужденных состояний доноров мышьяка в германии при спонтанном излучении акустических фононов в зависимости от одноосной деформации сжатия кристалла в направлении [110]. Вычислена зависимость от величины деформации населенностей примесных состояний, на переходах между которыми, как ожидается, возможна генерация стимулированного излучения при фотовозбуждении донорных центров.

\section{2. Теоретическая модель и результаты}

В недеформированном кристалле состояния зоны проводимости германия имеют четырехкратное вырож- дение, связанное с числом минимумов (долин) зоны проводимости в пространстве волновых векторов. Возбужденные состояния мелких доноров, которые мало подвержены влиянию короткодействующего потенциала центральной ячейки, также участвуют в проводимости кристалла германия. При одноосной деформации кристалла вдоль направления [110] происходит расщепление состояний зоны проводимости с разделением на пары долин. Это же происходит и с состояниями донорных центров [3]. Цифрами на рис. 1 выделены пары долин, на которые происходит разделение, вызванное деформацией в этом направлении. На рис. 2

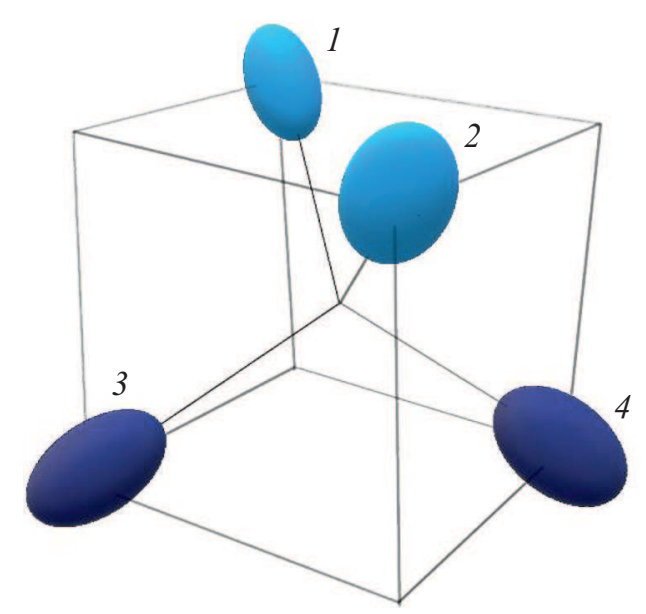

Рис. 1. Схема долин зоны проводимости кристалла германия. Цифрами выделены пары долин $((1,2)$ и $(3,4))$, на которые происходит расщепление состояний зоны проводимости и состояний мелких доноров при деформации кристалла в направлении $\{110\}$. 


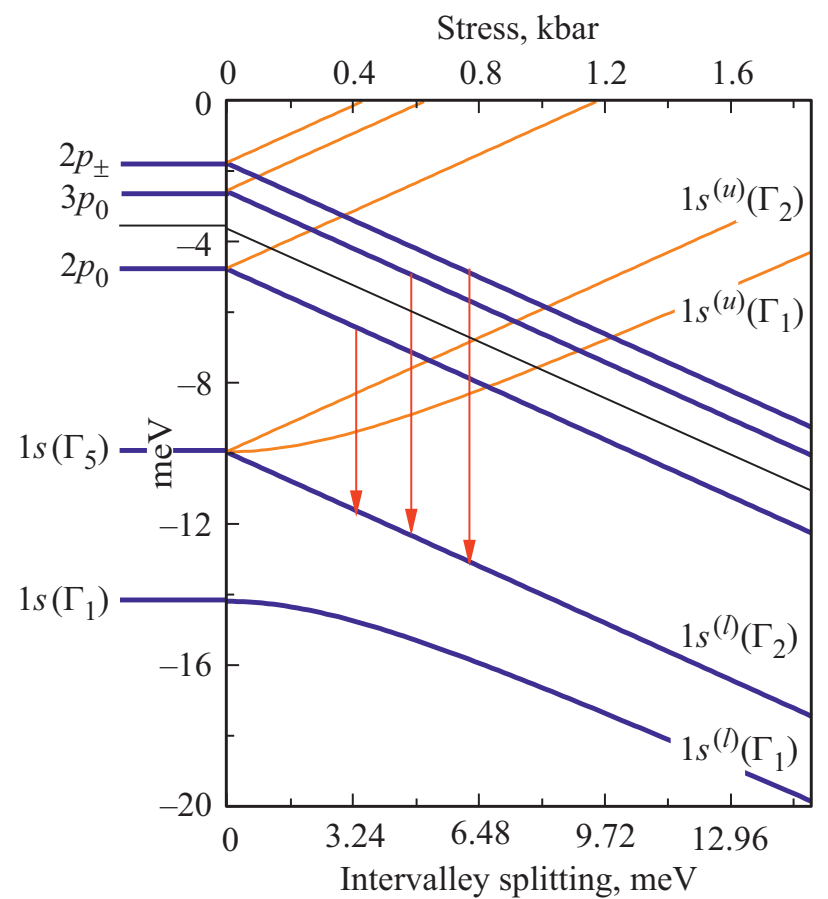

Рис. 2. Схема уровней донора мышьяка в германии в зависимости от деформации сжатия в направлении [110]. Стрелками указаны оптически разрешенные переходы, на которых возможно формирование инверсии.

представлена схема уровней донора мышьяка в германии в зависимости от одноосной деформации сжатия кристалла в направлении [110]. Индексы $(l)$ и $(u)$ в обозначениях $1 s$-состояний при деформации кристалла означают принадлежность состояния верхней (upper) или нижней (low) группе долин. Стрелками указаны оптически разрешенные переходы в электродипольном приближении, на которых, как ожидается, возможно формирование инверсной населенности при оптическом возбуждении донорных центров, а следовательно, и генерации стимулированного излучения в ТГц диапазоне частот. Полагается, что инверсия населенностей, достаточная для генерации стимулированного излучения, более легко достижима на состояниях донорного центра, связанных с нижней парой долин, образующихся при деформации кристалла, поэтому в данной работе выполнен расчет скоростей релаксации именно этой группы состояний. Интерес к вычислению темпов релаксации состояний донора в зависимости деформации сжатия кристалла от направления [110] связан с тем, что при таком направлении деформации сохраняется возможность реализации четырехуровневой лазерной схемы на внутрицентровых переходах мелких доноров в отличие от случая деформации вдоль направления [111].

Вычисление скоростей безызлучательной релаксации мелких доноров в кристалле германия производилось в рамках той же модели, что и в работе [1], с использованием тех же пробных огибающих волновых функций, за исключением способа определения параметров волновой функции $1 s$-состояний, задающих ее локализацию в пространстве. В отличие от подхода в работе [1], где эти параметры определены на основе поведения асимптотики волновой функции на большом удалении от примесного центра, в настоящей работе для возбужденных 1s-состояний эти параметры вычислены на основе вариационной процедуры минимизации энергии состояния с гамильтонианом эффективных масс и кулоновским потенциалом примесного центра [3]. Для основного состояния значения этих параметров уменьшены в $\sqrt{E_{2} / E_{1}}$ раз, где $E_{1}$ и $E_{2}$ - энергии основного и первого возбужденного $1 s$-состояний соответственно. Другими словами, для основного состояния изменена локализация волновой функции в пространстве в соответствии с зависимостью от энергии состояния асимптотического поведения волновой функции на большом удалении от центра [4], но с сохранением той же анизотропии. Кроме того, полагалось, что значения этих радиусов локализации не зависят от деформации кристалла. Такой подход приводит к несколько меньшим значениям темпов релаксации возбужденных состояний на переходе в состояния $1 s$, по сравнению с темпами тех же переходов в работе [1].

На рис. $3, a-c$ показаны зависимости от давления темпов ключевых переходов, определяющих населенности состояний, на которых, как ожидается, возможно формирование инверсной населенности. Как видно, скорость внутрицентровых переходов с излучением акустических фононов сильно зависит от приложенной деформации. Такая зависимость обусловлена двумя факторами, один из которых - изменение энергии переходов, второй изменение долинных вкладов в волновую функцию состояния донора. Зависимости энергий состояний доноров и долинной структуры их волновых функций взяты из работы [5].

На основе вычисленных времен в рамках балансных уравнений рассчитаны населенности состояний донора мышьяка в условиях фотоионизации доноров в зависимости от приложенного давления в кристаллографическом направлении [110]. Используемая система балансных уравнений включает в себя состояния, связанные как с нижними, так и с верхними долинами зоны проводимости германия. Всего система включала 12 уровней: все состояния от основного до состояния $2 p_{ \pm}$и эффективный уровень, связанный с зоной проводимости и высоковозбужденными состояниями донорного центра, лежали по энергии выше уровня $2 p_{ \pm}$. Расчеты выполнены при учете только внутридолинных переходов между состояниями донорного центра с излучением фононов и в пренебрежении междолинными процессами электронфононного взаимодействия. Значение темпа фотоионизации было взято $5 \cdot 10^{9} \mathrm{c}^{-1}$. Для описания захвата носителей из зоны проводимости использовался такой же подход, как и в работе [1]. Захват происходит в узкий энергетический поясок связанных состояний под самым дном зоны проводимости с темпом $2 \cdot 10^{9} \mathrm{c}^{-1}$. Все эти 

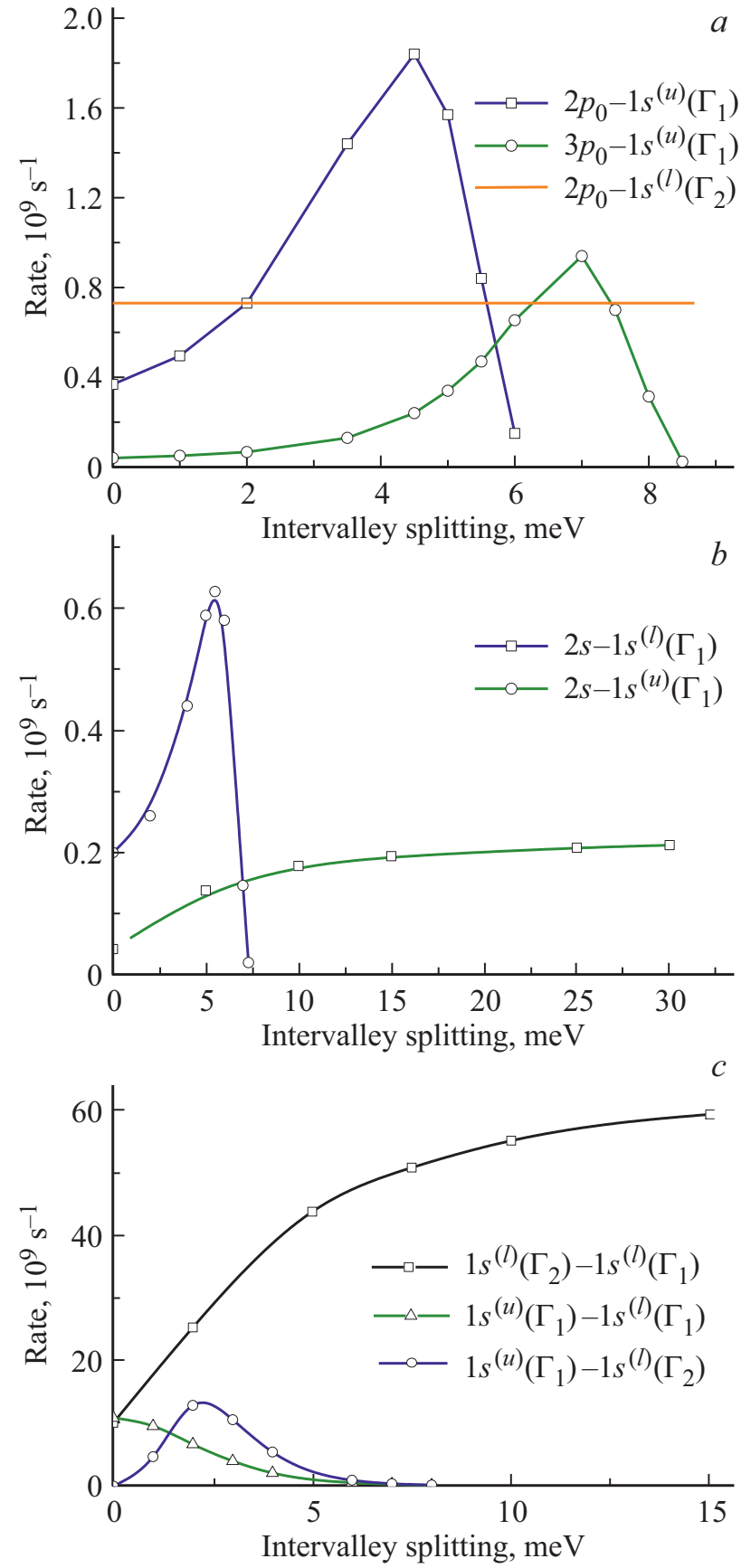

Рис. 3. $a-c-$ зависимости от деформации темпов ключевых переходов, определяющих населенности состояний донора мышьяка в германии при оптическом возбуждении. Давление 1 кбар в случае сжатия кристалла вдоль направления [110] соответствует междолинному расщеплению $\sim 8.1$ мэВ.

состояния имеют разную топологию волновой функции. Далее происходит релаксация по состояниям примесного центра, которая осуществляется не каскадным образом: переходы с излучением фононов при прочих равных условиях с большей вероятностью происходят между состояниями с близкой топологией огибающих волновых функций, определяемых квантовыми числами $n, l, m$. Это приводит к тому, что в процессе релаксации возникают различные потоки носителей по различным маршрутам в „лестнице“ энергетических уровней. Доля носителей, проходящих через заданный уровень, соответствующий состояниям $2 p_{0}, 2 s, 3 p_{0}$ и $2 p_{ \pm}$, зависит от того, каким образом распределяются носители по состояниям с различными топологиями волновых функций в узком пояске энергий связанных состояний под самым дном зоны проводимости, куда непосредственно осуществляется захват. Полагается, что носители по таким состояниям распределяются равномерно. Таким образом, можно положить, что эффективно захват осуществляется с равным темпом в состояния $2 p_{0}, 2 s, 3 p_{0}$ и $2 p_{ \pm}$, суммарное значение которых равняется общему темпу захвата $2 \cdot 10^{9} \mathrm{c}^{-1}$.

На рис. 4 представлена рассчитанная зависимость от деформации населенностей состояний донора, на переходах между которыми, как предполагается, возможно достижение эффекта стимулированного излучения. Как видно из рис. 4, заметная инверсная населенность формируется на разрешенных в дипольном приближении переходах $2 p_{0}-1 s^{(l)}\left(\Gamma_{2}\right)$ и $2 p_{ \pm}-1 s^{(l)}\left(\Gamma_{2}\right)$, которая зависит от величины приложенного давления. В отличие от проведенных ранее расчетов [1], в которых при нулевой деформации кристалла максимальное значение инверсии населенностей достигалось на переходе $2 p_{ \pm}-1 s\left(\Gamma_{5}\right)$, здесь верхним уровнем рабочего перехода с большей вероятностью становится $2 p_{0}$ даже в недеформированных образцах. Такое отличие обусловлено упомянутой выше модификацией модели расчета, а именно изменением способа вычисления параметров, определяющих локализацию волновых функций $1 s$-состояний доноров.

Следует отметить, что мышьяк в германии является мелким донором, и энергетический зазор между основным и первым возбужденным состояниями $\sim 5$ мэВ. Это приводит к тому, что термическая населенность

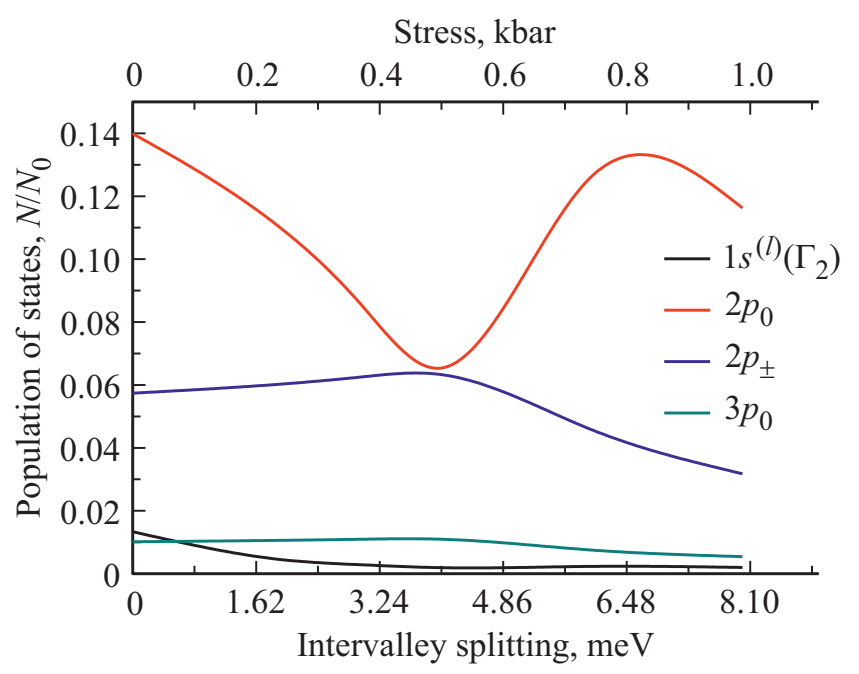

Рис. 4. Зависимость от приложенного давления населенностей уровней донора мышьяка, связанных с нижними долинами зоны проводимости, в германии. $N_{0}-$ концентрация доноров. 
первого возбужденного $1 s$-состояния, соответствующего нижнему уровню ожидаемого рабочего перехода, при температуре $\sim 15 \mathrm{~K}$ сравнивается с рассчитанной населенностью $2 p$-уровней. Это обстоятельство накладывает значительные ограничения на рабочие температуры и серьезные требования к организации хорошего теплоотвода от потенциального лазера на основе германия, так как даже в условиях охлаждения образца жидким гелием происходит его нагрев излучением накачки.

\section{3. Заключение}

Теоретически показанная возможность формирования инверсной населенности уровней донора мышьяка в кристалле германия при оптическом возбуждении среды дает основания для экспериментальной проверки возможности генерации стимулированного излучения на внутрицентровых переходах мелких доноров. Одноосная деформация в кристаллографическом направлении [110] позволяет изменять скорости релаксации возбужденных состояний, что приводит к незначительному изменению инверсии населенностей на переходе $2 p_{ \pm}-1 s^{(l)}\left(\Gamma_{2}\right)$ и немонотонной зависимости от приложенного давления инверсии населенностей на переходе $2 p_{0}-1 s^{(l)}\left(\Gamma_{2}\right)$. В отсутствие одноосного сжатия кристалла ожидается, что генерация будет развиваться на переходе $2 p_{0}-1 s^{(l)}\left(\Gamma_{2}\right)$. При давлении $\sim 0.5$ кбар происходит выравнивание населенностей состояний $2 p_{0}$ и $2 p_{ \pm}$, следовательно, за счет того, что матричный элемент оптического перехода $2 p_{ \pm}-1 s^{(l)}\left(\Gamma_{2}\right)$ в электродипольном приближении выше, чем перехода $2 p_{0}-1 s^{(l)}\left(\Gamma_{2}\right)$, будет происходить переключение рабочего перехода с $2 p_{0}-1 s^{(l)}\left(\Gamma_{2}\right)$ на $2 p_{ \pm}-1 s^{(l)}\left(\Gamma_{2}\right)$ и, следовательно, изменение частоты стимулированного излучения в ТГц диапазоне частотного спектра.

Для экспериментального наблюдения эффекта стимулированного излучения при оптическом возбуждении среды необходимо обеспечить низкую температуру кристалла $(<15 \mathrm{~K})$. Для этого видится возможным охлаждение образцов жидким гелием и использование импульсного оптического возбуждения. Необходимость импульсной оптической накачки связана с тем, что, с одной стороны, нужно обеспечить достаточное время возбуждения для развития генерации, а с другой не допустить перегрева образца. Предполагается, что возбуждение среды возможно осуществлять с помощью импульсного $\mathrm{CO}_{2}$-лазера.

\section{Финансирование работы}

Работа выполнена при поддержке Российского фонда фундаментальных исследований (№ 18-42-520064).

\section{Конфликт интересов}

Авторы заявляют, что у них нет конфликта интересов.

\section{Список литературы}

[1] В.В. Цыпленков, В.Н. Шастин. ФТП, 52, 1469 (2018).

[2] Р.Х. Жукавин, К.А. Ковалевский, Ю.Ю. Чопорова, В.В. Цыпленков, В.В. Герасимов, П.А. Бушуйкин, Б.А. Князев, Н.В. Абросимов, С.Г. Павлов, Г.-В. Хьюберс, В.Н. Шастин. Письма ЖЭТФ, 110, 677 (2019).

[3] R.J. Bell, W.T. Bousman,jr., G.M. Goldman, D.G. Rathbun. Surf. Sci., 7, 293 (1967).

[4] Б.И. Шкловский, А.Л. Эфрос. Электронные свойства легированных полупроводников (М., Наука, 1979) с. 38.

[5] A. Kobayashi, K. Suzuki. Phys. Status Solidi B, 98, 643 (1980).

Редактор Г.А. Оганесян

\section{Influence of uniaxial stress along [110] direction on relaxation of arsenic shallow donor states in germanium}

\section{V.V. Tsyplenkov, V.N. Shastin}

Institute for Physics of Microstructures, Russian Academy of Sciences, 603950 Nizhny Novgorod, Russia

\begin{abstract}
Abstract Analysis of acoustical phonon assisted relaxation rates of arsenic donor states has been carried out in depends on uniaxial compressive stress of crystal along [110] direction under low temperature $(<10 \mathrm{~K})$. As shown, under optical excitation the inversion population of donor energy levels is formed that depends on deformation of crystal. This give grounds to suppose that stimulated emission on arsenic shallow donor intracenter transitions in $\mathrm{THz}$ range is possible under optical excitation. As shown, uniaxial stress along [110] direction can result to switch laser transition and stimulated emission frequency.
\end{abstract}

